\title{
JOGOS DIGITAIS EDUCACIONAIS: BENEFÍCIOS E DESAFIOS
}

\author{
Rafael Savi ${ }^{1}$ \\ Programa de Pós-Graduação em Engenharia e Gestão do Conhecimento / UFSC
}

\author{
Vania Ribas Ulbricht, Dra ${ }^{2}$ \\ Programas de Pós-Graduação em Engenharia e Gestão do Conhecimento / UFSC e de \\ Design /AM
}

Resumo: As instituições de ensino estão ampliando o uso das tecnologias de informação e comunicação para oferecer aos alunos mídias interativas que possam enriquecer as aulas. Os jogos digitais aparecem nesse contexto como um recurso didático que contém características que podem trazer uma série de benefícios para as práticas de ensino e aprendizagem. Este artigo apresenta o potencial dos jogos digitais educacionais, baseado em estudos de especialistas, trás exemplos de jogos utilizados em diferentes níveis de ensino e aponta problemas que ainda precisam ser superados para facilitar o emprego dos jogos digitais educacionais.

Palavras-chave: jogos educacionais; tecnologias educacionais; jogos sérios.

\section{DIGITAL EDUCATIONAL GAMES: BENEFITS AND CHALLENGES}

\begin{abstract}
Educational institutions are expanding the usage of information and communication technology to offer interactive media and enhanced classes for their students. In this context, digital games appear as a didactic resource with characteristics that bring many benefits for educational practices. This paper presents educational games potentiality, based on a review of experts studies, shows examples of games used at different levels of education and points problems that still need to be overcome to facilitate the usage of educational games.
\end{abstract}

Keywords: educational games; educational technology; serious games.

\section{Introdução}

Os jogos de vídeo games e computadores conquistaram um espaço importante na vida de crianças, jovens e adultos e hoje é um dos setores que mais cresce na indústria de mídia e entretenimento. Estudos recentes da consultoria PricewaterhouseCoopers estimam que em 2008 o faturamento do mercado de jogos deverá superar o do setor de música, que sempre teve destaque econômico (Marketing Charts, 2008).

Com um faturamento bilionário os jogos digitais já assumiram um papel de destaque na cultura contemporânea, levando diversos pesquisadores a desenvolverem estudos para

\footnotetext{
${ }^{1}$ rafaelsavi@yahoo.com.br
}

2 ulbricht@floripa.com.br 
entender porque os jogos digitais são tão atraentes e quais impactos causam na vida das pessoas (Kirriemuir; Mcfarlane, 2004).

Muitos jovens seduzidos pelos jogos digitais permanecem longos períodos totalmente empenhados nos desafios e fantasias destes artefatos de mídia, dando a impressão de que são imunes a distrações e que nada é capaz de desconcentrá-los.

Mas os jogos digitais costumam absorver muitas horas dos jogadores e consomem um tempo que poderia ser aproveitado em outras atividades, como o estudo, por exemplo. Isto gera reclamações entre pais e professores, pois gostariam que seus filhos e alunos aplicassem nos estudos o mesmo nível de atenção e comprometimento dedicado aos jogos (Kirriemuir; Mcfarlane, 2004).

Conseguir desviar a atenção que os estudantes dão aos jogos para atividades educacionais não é tarefa simples. Por isso, tem aumentado o número de pesquisas que tentam encontrar formas de unir ensino e diversão com o desenvolvimento de jogos educacionais. Por proporcionarem práticas educacionais atrativas e inovadoras, onde $\mathrm{o}$ aluno tem a chance de aprender de forma mais ativa, dinâmica e motivadora, os jogos educacionais podem se tornar auxiliares importantes do processo de ensino e aprendizagem.

Mas para serem utilizados com fins educacionais os jogos precisam ter objetivos de aprendizagem bem definidos e ensinar conteúdos das disciplinas aos usuários, ou então, promover o desenvolvimento de estratégias ou habilidades importantes para ampliar a capacidade cognitiva e intelectual dos alunos (Gros, 2003).

Durante muitos anos se discutiu a possibilidade dos vídeo games influenciarem negativamente os jogadores e estimularem a violência em crianças e adolescentes. Nos últimos anos, porém, aumentou o interesse para a pesquisa dos aspectos positivos dos jogos, seus benefícios para os jogadores, potencialidades como recurso didático e uso na educação (Eck, 2006).

Agora, ao invés das instituições de ensino fecharem as portas para os jogos, existe um crescente interesse entre pesquisadores e professores em descobrir de que formas os jogos digitais podem ser usados como recurso para apoiar a aprendizagem e quais são os seus benefícios (Kirriemuir; Mcfarlane, 2004).

\section{As Potencialidades dos Jogos Digitais Educacionais}

Uma das principais formas de acesso ao mundo da tecnologia para crianças e jovens é o jogo digital, pois geralmente o primeiro contato com equipamentos eletrônicos acontece por meio de um vídeo game (Gros, 2003).

Os jogos digitais podem ser definidos como ambientes atraentes e interativos que capturam a atenção do jogador ao oferecer desafios que exigem níveis crescentes de destreza e habilidades (Balasubramanian; Wilson, 2006).

Balasubramanian e Wilson (2006), com base em estudos de Glazier (1973), Prensky (2001) e Rasmusen (2001), apontam os componentes básicos dos jogos digitais, que são: 1) o papel ou personagem do jogador; 2) as regras do jogo; 3) metas e objetivos; 4) quebra-cabeças, problemas ou desafios; 5) história ou narrativa; 6) interações do jogador; 7) estratégias; 8) feedback e resultados.

Mas para serem utilizados como instrumentos educacionais os jogos devem conter ainda algumas características específicas para atender as necessidades vinculadas à 
aprendizagem. Por isso os softwares educacionais, entre eles os jogos, "devem possuir objetivos pedagógicos e sua utilização deve estar inserida em um contexto e em uma situação de ensino baseados em uma metodologia que oriente o processo, através da interação, da motivação e da descoberta, facilitando a aprendizagem de um conteúdo" (Prieto et al., 2005, p. 10).

Quando preparados para o contexto educacional os jogos digitais podem receber diferentes nomenclaturas. As mais comuns são jogos educacionais ou educativos, jogos de aprendizagem ou jogos sérios (serious games), sendo que alguns tipos de simuladores também podem ser considerados jogos educacionais.

Normalmente, quando se divulga a utilização de jogos educacionais, há um destaque para o poder motivador dessa mídia. Mas o potencial deles vai muito além do fator "motivação", pois ajudam os estudantes a desenvolverem uma série de habilidades e estratégias e, por isso, começam a ser tratados como importantes materiais didáticos (Gros, 2003).

A seguir são elencados alguns benefícios que os jogos digitais educacionais podem trazer aos processos de ensino e aprendizagem:

- Efeito motivador: Os jogos educacionais demonstram ter alta capacidade para divertir e entreter as pessoas ao mesmo tempo em que incentivam o aprendizado por meio de ambientes interativos e dinâmicos (Hsiao, 2007). Conseguem provocar o interesse e motivam estudantes com desafios, curiosidade, interação e fantasia (Balasubramanian; Wilson, 2006).

As tecnologias dos jogos digitais proporcionam uma experiência estética visual e espacial muito rica e, com isso, são capazes de seduzir os jogadores e atraí-los para dentro de mundos fictícios que despertam sentimentos de aventura e prazer (Mitchell; Savill-Smith, 2004).

Ter componentes de prazer e diversão inseridos nos processos de estudo é importante porque, com o aluno mais relaxado, geralmente há maior recepção e disposição para o aprendizado (Prensky, 2001; Hsiao, 2007). Jogos bem projetados levam os jogadores para um estado de intensa concentração e envolvimento entusiasmado (chamado de estado de fluxo), onde a ânsia por vencer promove o desenvolvimento de novas habilidades (Mitchell; Savill-Smith, 2004).

As metas e desafios que precisam ser vencidos nos jogos geram provocações nas pessoas, mantendo-as motivadas e, em alguns casos, podem até recuperar o ânimo de quem perdeu o interesse pelo estudo (Ritchie; Dodge, 1992).

- Facilitador do aprendizado: Jogos digitais têm a capacidade de facilitar o aprendizado em vários campos de conhecimento.

Eles viabilizam a geração de elementos gráficos capazes de representar uma grande variedade de cenários. Por exemplo, auxiliam o entendimento de ciências e matemática quando se torna difícil manipular e visualizar determinados conceitos, como moléculas, células e gráficos matemáticos (Fabricatore, 2000; Mitchell; Savill-Smith, 2004).

Os jogos colocam o aluno no papel de tomador de decisão e o expõe a níveis crescentes de desafios para possibilitar uma aprendizagem através da tentativa e erro (Mitchell; Savill-Smith, 2004). 
Projetistas de jogos inserem o usuário num ambiente de aprendizagem e então aumentam a complexidade das situações e, à medida que as habilidades melhoram, as reações do jogador se tornam mais rápidas e as decisões são tomadas com maior velocidade (Kirriemuir; Mcfarlane, 2004).

Muitos professores reconhecem que os jogos, além de facilitarem a aquisição de conteúdos, contribuem também para o desenvolvimento de uma grande variedade de estratégias que são importantes para a aprendizagem, como resolução de problemas, raciocínio dedutivo e memorização (Mcfarlane; Sparrowhawk; Heald, 2002). Outros benefícios dos jogos e simuladores incluem a melhoria do pensamento estratégico e insight, melhoria das habilidades psicomotoras, desenvolvimento de habilidades analíticas e habilidades computacionais (Mitchell; Savill-Smith, 2004). Alguns jogos online, que são disputados em equipes, ajudam a aprimorar o desenvolvimento de estratégias em grupo e a prática do trabalho cooperativo (Gros, 2003).

- Desenvolvimento de habilidades cognitivas: Os jogos promovem o desenvolvimento intelectual, já que para vencer os desafios o jogador precisa elaborar estratégias e entender como os diferentes elementos do jogo se relacionam (Gros, 2003). Também desenvolvem várias habilidades cognitivas, como a resolução de problemas, tomada de decisão, reconhecimento de padrões, processamento de informações, criatividade e pensamento crítico (Balasubramanian; Wilson, 2006).

- Aprendizado por descoberta: Desenvolvem a capacidade de explorar, experimentar e colaborar (Becta, 2001), pois o feedback instantâneo e o ambiente livre de riscos provocam a experimentação e exploração, estimulando a curiosidade, aprendizagem por descoberta e perseverança (Mitchell; Savill-Smith, 2004).

- Experiência de novas identidades: Oferecem aos estudantes oportunidades de novas experiências de imersão em outros mundos e a vivenciar diferentes identidades. Por meio desta imersão ocorre o aprendizado de competências e conhecimentos associados com as identidades dos personagens dos jogos (Hsiao, 2007). Assim, num jogo ou simulador em que o estudante controla um engenheiro, médico ou piloto de avião, estará enfrentando os problemas e dilemas que fazem parte da vida destes profissionais e assimilando conteúdos e conhecimentos relativos às suas atividades.

- Socialização: Outra vantagem dos jogos educacionais é que eles também podem servir como agentes de socialização à medida que aproximam os alunos jogadores, competitivamente ou cooperativamente, dentro do mundo virtual ou no próprio ambiente físico de uma escola ou universidade. Em rede, com outros jogadores, os alunos têm a chance de compartilhar informações e experiências, expor problemas relativos aos jogos e ajudar uns aos outros, resultando num contexto de aprendizagem distribuída (Hsiao, 2007).

- Coordenação motora: Diversos tipos de jogos digitais promovem o desenvolvimento da coordenação motora e de habilidades espaciais (Gros, 2003).

- Comportamento expert: Crianças e jovens que jogam vídeo games se tornam experts no que o jogo propõe. Isso indica que jogos com desafios educacionais podem ter o potencial de tornar seus jogadores experts nos temas abordados (Vandeventer; White, 2002) 
Embora seja difícil encontrar em um único jogo todas as potencialidades apresentadas acima, procurou-se demonstrar como este tipo de mídia pode trazer uma série de benefícios ao ser utilizada como recurso didático nas práticas de ensino.

\section{Exemplos do Uso de Jogos Digitais Educacionais}

Os jogos digitais já são utilizados em diferentes níveis de ensino, indo desde a préescola até cursos de graduação, especializações e cursos corporativos. Esta seção irá abordar alguns exemplos em diferentes contextos.

Existem jogos que promovem ensinamentos básicos sobre linguagem e lógica para crianças que ainda estão em processo de alfabetização ou não sabem ler. Ao mesmo tempo, servem para ensinar os alunos a começarem a utilizar o computador e a desenvolverem coordenação com o mouse e teclado. Esses jogos são simples e abordam o reconhecimento de letras e números, ensino de melodias em instrumentos virtuais, desafios de lógica e memória e jogos de línguas. Exemplos desse tipo são os programas Gcompris $^{3}$ e Childsplay ${ }^{4}$, que apresentam conjuntos de jogos pré-escolares e de ensino fundamental. São softwares open source que podem ser livremente instalados e distribuídos. Há também portais web como o IGuinho ${ }^{5}$ e Discovery Kids ${ }^{6}$, similares aos exemplos anteriores, mas que disponibilizam os jogos na web.

Com algumas buscas na internet é possível encontrar muitas fontes de jogos educacionais ou simuladores. Um exemplo é o site do professor Romero Tavares da Silva $^{7}$, da Universidade Federal de Pernambuco - UFPE, que disponibiliza um conjunto de simuladores de física aplicáveis ao ensino médio. São abordados temas como movimento retilíneo, energia, ondas e termodinâmica em pequenas aplicações interativas que permitem ao usuário inserir diferentes valores para variáveis e acompanhar o resultado nas animações do simulador. Ao final do procedimento pode-se verificar os resultados das experiências em gráficos que são gerados automaticamente.

Alguns jogos abordam a saúde e bem estar como temática central, são os health games. Um exemplo deste gênero é o Escape from Diab ${ }^{8}$, jogo em fase final de desenvolvimento, que tem o objetivo de prevenir a obesidade infantil e a diabetes, direcionando a atenção das crianças para a importância de uma alimentação saudável e a prática de exercícios. Neste jogo, o usuário toma decisões e aprende como suas ações afetam a saúde dos personagens enquanto combate o vilão Rei Etes, que eliminou todas as oportunidades de exercícios de uma cidade e fornece quantidades ilimitadas de junk food para a população. Manter um estilo de vida saudável é a única maneira de vencer o jogo e derrotar o Rei Etes e seus guardas 9 .

O Escape from $\operatorname{Dia}^{10} b$ está sendo produzido por uma empresa americana em colaboração com o Children's Nutrition Research Center da Faculdade Baylor de Medicina, localizada em Houston, EUA.

\footnotetext{
${ }^{3}$ http://gcompris.net/-pt-br-

${ }^{4}$ http://childsplay.sourceforge.net/

${ }^{5}$ http://iguinho.ig.com.br/

${ }^{6} \mathrm{http} / / / \mathrm{www}$.discoverykidsbrasil.com/jogos/

${ }^{7} \mathrm{http} / / / \mathrm{www}$. fisica.ufpb.br/\% 7Eromero/

${ }^{8} \mathrm{http}: / /$ www.escapefromdiab.com/

${ }^{9} \mathrm{http}: / / \mathrm{www}$. treehugger.com/files/2007/12/escape_from_dia.php

${ }^{10} \mathrm{http}: / / \mathrm{www}$.archimageonline.com/diab.cfm
} 
Outro exemplo que aborda questões relacionadas com a saúde é $R e$-Mission ${ }^{11}$, um jogo para jovens com câncer que procura ser tanto divertido como informativo e, em vinte níveis, esclarece para os pacientes os diferentes tipos de tratamento, como eles funcionam, e a importância de realizá-los. O jogador controla um nano-robô que é injetado dentro dos corpos de pacientes e luta contra as infecções celulares do câncer. Além disso, deve monitorar a saúde do paciente virtual e informar seus sintomas para a médica dentro do jogo. Re-Mission foi criado pela organização sem fins lucrativos HopeLab $^{12}$ e está disponível gratuitamente no site oficial do jogo.

Também existem jogos que abordam questões políticas e sociais. O Global Conflicts: Palestine $^{13}$ é uma aventura com elementos de RPG que coloca o jogador no papel de um jornalista freelancer recém chegado a Jerusalém e que precisa produzir artigos sobre o conflito entre Israel e Palestina. Este jogo possibilita que as pessoas interajam com os dois lados da guerra e pode ser utilizado para o ensino de disciplinas como história, cidadania e ciências sociais enquanto referencia temas como direitos humanos, terrorismo e o funcionamento da mídia.

Já o $3 r d$ World Farmer ${ }^{14}$ desafia o jogador a manter sua família viva enquanto gerencia uma fazenda em área de conflito e pobreza na África. Deve-se investir todas as economias na plantação e torcer por resultados positivos, mas dificuldades climáticas, sociais e econômicas freqüentemente atingem famílias inteiras. Segundo seu criador, um estudante da Universidade de Tecnologia da Informação de Copenhagen, o jogo é difícil e as regras propositalmente não são justas, visando levar o jogador a experimentar situações de injustiças ao invés de apenas fazê-lo ouvir sobre elas, estimulando uma reflexão mais profunda sobre as condições de vida pela qual parte da população do planeta passa. O jogo pode ser utilizado em salas de aula para cada aluno jogar, refletir e depois discutir os assuntos abordados nas partidas com toda a classe.

Cursos de graduação e especialização também já estão utilizando jogos como material didático. Um exemplo é o Innova8, jogo 3D desenvolvido pela IBM para ajudar estudantes universitários e alunos de MBA a desenvolverem habilidades de negócios e aprenderem sobre tecnologias de informação. O jogo coloca o estudante dentro de um ambiente empresarial para visualizar como a tecnologia e as estratégias de negócios afetam o desempenho de uma organização. Os jogadores podem analisar os processos da empresa, identificar gargalos e explorar cenários what- If antes de implementar determinadas tecnologias. O jogo foi projetado para durar em torno de 1 hora em laboratórios como complemento de disciplinas de Gestão de Processos de Negócios, Estratégia Empresarial e Gestão de TI. (IBM Press release ${ }^{15}$ ).

Os jogos digitais também são uma estratégia para tornar os treinamentos das empresas mais interessantes e preparar funcionários para certificações. A empresa HP, por exemplo, utiliza jogos como meio de ensino para suas equipes de gestão de TI aprenderem sobre incidentes e problemas, planos de contingência, centros de dados, entre outros assuntos. $\mathrm{O}$ treinamento acontece por meio de uma corrida automobilística virtual onde a equipe da empresa tem quatro carros competindo contra outros vinte, e cada um deles possui treze sistemas complexos de TI, como hidráulica, monitoramento de motor, controle de tração, etc. No meio da competição estes sistemas começam a

\footnotetext{
11 http://www.re-mission.net/

$12 \mathrm{http}: / /$ www.hopelab.org/

${ }^{13}$ http://gamesbrasil.uol.com.br/comentarios.php?numero=14529

${ }^{14} \mathrm{http} / / / \mathrm{www}$. socialimpactgames.com/modules.php?op=modload $\&$ name $=$ News $\&$ file $=$ article $\&$ sid $=279 \& \mathrm{~m}$ ode $=$ thread\&order $=1 \&$ thold $=0$

${ }^{15}$ http://www-03.ibm.com/press/us/en/pressrelease/22549.wss
} 
apresentar defeitos e o piloto virtual relata a situação para um help desk, que precisa resolver a situação. Se a equipe não for ágil e algum carro for ultrapassado há uma penalidade para a empresa, que perde pontos e patrocínios ${ }^{16}$.

Outro jogo para ambientes corporativos é o Virtual Leader, focado na capacitação de lideranças em uma série de cenários que simulam reuniões com diversos objetivos e agendas. Nele pode-se observar os participantes das reuniões e avaliar o nível de humor e linguagem corporal de cada personagem, entre outras características, enquanto idéias são discutidas. O jogador deve ter suas idéias aceitas sem causar mal estar ou conflitos nos outros participantes das reuniões virtuais. Neste jogo são trabalhadas habilidades para aprender a influenciar outras pessoas, gerenciar conflitos, criação de equipes, colaboração e motivação.

Esses exemplos ilustram a abrangência e potencial do uso dos jogos digitais como material de ensino e aprendizagem. Cabe comentar que jogos educacionais em língua portuguesa ainda são raros e a maioria deste tipo de conteúdo ainda está disponível somente em inglês.

\section{Problemas e Desafios}

Apesar do potencial e benefícios, os jogos digitais educacionais ainda são pouco empregados e, para muitos professores, encontrar e utilizar bons jogos continua sendo um desafio (Balasubramanian; Wilson, 2006).

Isso ocorre, em boa parte, porque muitos jogos educacionais têm feito uso limitado de princípios pedagógicos e acabam sendo ignorados pelos educadores por agregarem pouco valor às aulas. Nesse sentido, Van Eck (2006) comenta que empresas e especialistas no desenvolvimento de games, que não possuem conhecimentos específicos sobre teoria e prática do uso de jogos em ambientes de aprendizagem, criam produtos educacionais que são atraentes e divertidos, mas falham em relação aos objetivos de aprendizagem. Por outro lado, jogos desenvolvidos por educadores com um viés mais acadêmico, com pouco conhecimento da arte, ciência e cultura de projetos de jogos, na maioria dos casos resultam em artefatos pouco divertidos que não conseguem atrair a atenção dos alunos.

Os jogos educacionais devem atender a requisitos pedagógicos, mas também é preciso tomar cuidado para não tornar o jogo somente um produto didatizado, fazendo-o perder seu caráter prazeroso e espontâneo (Fortuna, 2000). É necessário encontrar a sinergia entre pedagogia e diversão nos jogos educacionais, mas isso tem demonstrado ser uma tarefa difícil.

Atualmente muitos jogos e softwares educacionais não atingem as expectativas dos educadores e alunos e algumas das principais razões são listadas a seguir (Becta, 2001; Kirriemuir; Mcfarlane, 2004):

- A maioria dos jogos educacionais é muito simples em relação aos vídeo games comerciais de competição e não atendem as expectativas dos alunos mais exigentes, já acostumados com a sofisticação dos jogos de entretenimento;

- As tarefas propostas são repetitivas, por exemplo, efetuar somas ou exercitar a memória continuamente, de forma que o jogo se torne chato muito cedo;

\footnotetext{
${ }^{16}$ http://computerworld.uol.com.br/governanca/2007/04/04/idgnoticia.2007-04-04.4742287204/
} 
- As tarefas são muito pobres e não possibilitam uma compreensão progressiva dos conteúdos;

- A diversidade de atividades é severamente limitada dentro do jogo, normalmente concentrando o aprendizado numa única habilidade, ou então, na acumulação de conteúdos homogêneos.

- O conteúdo e estilo de muitos jogos são projetados para alunos do sexo masculino, o que pode fazer com que as alunas não se sintam interessadas e não se engajem nos objetivos de aprendizagem propostos;

- A existência de requisitos técnicos em alguns programas dificultam a execução, como a instalação de plugins ou módulos especiais;

- Em alguns casos, a linguagem de determinados jogos pode ser incompatível com a faixa etária dos alunos;

Além disso, várias questões como a relevância para currículo, precisão de conteúdos e compatibilidade da duração dos jogos com o horário de uso dos laboratórios de informática têm impedido que os jogos digitais educacionais se tornem uma atividade predominante nas escolas (Kirriemuir; Mcfarlane, 2004).

Muitas vezes, também há receio entre professores de que os alunos não vão participar ou cooperar, ou que as atividades com computadores exponham suas vulnerabilidades tecnológicas em relação ao conhecimento dos alunos que, muitas vezes, entendem mais de informática do que seus mestres (Balasubramanian; Wilson, 2006).

Saber como avaliar o progresso da aprendizagem dos alunos é outra questão que inibe o uso dos jogos pelos professores, especialmente no ensino on-line ou quando se tem classes com grande quantidade de alunos. Não basta apenas propor a atividade com jogos, é necessário verificar se os alunos estão atingindo os objetivos propostos e fornecer algum tipo de feedback para eles. Funcionalidades para o acompanhamento do progresso das turmas não são freqüentemente encontrados nos jogos educacionais, mas alguns automatismos podem ser auxiliares importantes para os professores, como por exemplo, a geração de relatórios informando em que nível cada aluno chegou, quanto tempo levou para resolver cada problema, principais dificuldades, erros cometidos, etc.

A criação de jogos educacionais mais sofisticados também é afetada pelos custos envolvidos no desenvolvimento, pois esse é um tipo de software complexo que normalmente demanda o emprego de várias áreas da computação, como banco de dados, redes de computadores, computação gráfica e estrutura de dados. Requer também a participação de artistas gráficos e músicos para a criação dos cenários, personagens, objetos e efeitos sonoros, e de especialistas nos conteúdos educacionais que o jogo pretende passar. Portanto, torna-se difícil desenvolver jogos educacionais com qualidade técnica, artística e pedagógica sem o envolvimento de uma equipe multidisciplinar para o projeto, que resulta em custos significativos para recursos humanos.

Nos jogos de entretenimento as questões técnicas e artísticas são tratadas com o auxílio de ferramentas de apoio ao desenvolvimento, que maximizam a produtividade dos desenvolvedores por meio de gerenciadores de algoritmos e com programas que facilitam a criação de personagens, cenários e objetos (Ambrosine, 2008). Além de facilitarem o desenvolvimento, o emprego desses aplicativos também pode reduzir custos. No entanto, ferramentas desse gênero focadas em jogos educacionais e que atendam a necessidades e requisitos pedagógicos ainda são raras e limitadas. 


\section{Considerações Finais}

Em um mundo com mídias cada vez mais sedutoras e atraentes, as salas de aula com quadro negro e giz estão se tornando lugares monótonos para os alunos acostumados ao dinamismo das buscas feitas na internet, com a velocidade das mensagens instantâneas e a versatilidade do telefone celular.

Jogos educacionais bem projetados podem ser criados e utilizados para unir práticas educativas com recursos multimídia em ambientes lúdicos a fim de estimular e enriquecer as atividades de ensino e aprendizagem. Os benefícios e potencialidades desse tipo de mídia são variados e continuam a ser estudados por educadores e pesquisadores. Desafios de ordem técnica e, principalmente pedagógicos, ainda precisam ser tratados para os jogos educacionais serem adotados com maior facilidade pelos professores como eficientes materiais didáticos.

Mas os exemplos da utilização de jogos por empresas, escolas e universidades já existem e estão aumentando. A tendência hoje é de que as tecnologias de informação ampliem a presença nas práticas de ensino e, nesse contexto, entende-se que os jogos digitais educacionais podem ser elementos importantes para enriquecer aulas e ambientes virtuais de aprendizagem.

\section{Referências}

AMBROSINE. Game Creation Resources. 2008. Disponível em:

$<$ http://www.ambrosine.com/resource.html>. Acesso em: 28 set. 2008.

BALASUBRAMANIAN, Nathan; WILSON, Brent G. Games and Simulations. In: SOCIETY FOR INFORMATION TECHNOLOGY AND TEACHER EDUCATION INTERNATIONAL CONFERENCE, 2006. Proceedings...v.1. 2006. Disponível em: $<\mathrm{http}$ ///site.aace.org/pubs/foresite/GamesAndSimulations1.pdf>. Acesso em: $23 \mathrm{dez}$. 2007.

BECTA. Computer Games in Education Project. Coventry: BECTA, 2001. Disponível em:<http://partners.becta.org.uk/index.php?section=rh\&rid=13595>. Acesso em 27 set. 2008.

ECK, Richard Van. Digital Game-Based Learning: It. Educase Review, v. 41, n. 2, p.16-30, mar. 2006. Disponível em:

<http://www.educause.edu/apps/er/erm06/erm0620.asp>. Acesso em: 20 out. 2006.

FABRICATORE, C. Learning and videogames: An unexploited synergy. In:

INTERNATIONAL CONFERENCE OF THE ASSOCIATION FOR EDUCATIONAL COMMUNICATIONS AND TECHNOLOGY, 2000, Denver, Colorado. Proceedings... Farmington Hills: Learning Development Institute, 2000.

FORTUNA, Tânia Ramos. Sala de aula é lugar de brincar? In: XAVIER, M.L.F. e DALLA ZEN, M.I.H. Planejamento: análises menos convencionais. Porto Alegre: Mediação, 2000 (Cadernos de Educação Básica, 6) p. 147-164

GLAZIER, Raymonf. How to design educational games: game design manual for teachers and curriculum developers. 4. ed. Cambridge: Abt Associates, 1973.

GROS, Begoña. The impact of digital games in education. First Monday, v. 8, n. 7, jul. 2003. Disponível em: 
<http://www.firstmonday.org/issues/issue8_7/xyzgros/index.html>. Acesso em: 22 out. 2007.

HSIAO, Hui-Chun. A Brief Review of Digital Games and Learning. DIGITEL 2007, The First IEEE International Workshop on Digital Game and Intelligent Toy Enhanced Learning. Los Alamitos, CA, USA: IEEE Computer Society, 2007. 124-129 p. Disponível em: <http://doi.ieeecomputersociety.org/10.1109/DIGITEL.2007.3>. Acesso em 06 jan. 2007.

KIRRIEMUIR, John; MCFARLANE, Angela. Literature Review in Games and Learning. Bristol: Futurelab, 2004. 39 p. Disponível em:

$<$ http://www.futurelab.org.uk/resources/publications_reports_articles/literature_reviews /Literature_Review378>. Acesso em 20 set. 2008.

MCFARLANE, Angela; SPARROWHAWK, Anne; HEALD Ysanne. Report on the educational use of games: An exploration by TEEM of the contribution which games can make to the education process. 2002. Disponível em:<http://www.teem.org.uk/publications/teem_gamesined_full.pdf $>$. Acesso em: 29 set. 2008.

MARKETING CHARTS. Videogames to Surpass Music in Revenue This Year. Disponível em: <http://www.marketingcharts.com/interactive/pwc-videogames-tosurpass-music-in-revenue-this-year-750/>. Acesso em: 20 set. 2008.

MITCHELL, Alice; SAVILL-SMITH, Carol. The use of computer and video games for learning: A review of the literature. Londres: Learning and Skills Development Agency (LSDA), 2004. Disponível em: <htttp://www.lsda.org.uk/files/PDF/1529.pdf>. Acesso em 20 set. 2008.

PRIETO, Lilian Medianeira et al. Uso das Tecnologias Digitais em Atividades Didáticas nas Séries Iniciais. Renote: revista novas tecnologias na educação, Porto Alegre, v. 3, n. 1, p.1-11, maio 2005. Disponível em: $<$ http://www.cinted.ufrgs.br/renote/maio2005/artigos/a6_seriesiniciais_revisado.pdf>. Acesso em: 26 maio 2007.

PRENSKY, Mark. Digital game-based learning. New York: McGraw-Hill, 2001.

RASMUSEN, Eric. Games and information: An introduction to game theory (3rd ed.). Malden: Blackwell, 2001.

RITCHIE, D.; DODGE, B. Integrating Technology Usage across the Curriculum through Educational Adventure Games. In: Anais... . p.10. Houston. Restaurado de http://eric.ed.gov/ERICWebPortal/custom/portlets/recordDetails/detailmini.jsp?_nfpb=t rue\&_\&ERICExtSearch_SearchValue_0=ED349955\&ERICExtSearch_SearchType_0= no\&accno=ED349955, 1992.

VANDEVENTER, Stephanie S.;WHITE, James A. Expert Behavior in Children's Video Game Play. Simulation Gaming, v. 33, n. 1, p. 28-48, 2002. Disponível em: <http://sag.sagepub.com/cgi/content/abstract/33/1/28>. Acesso em: 20 set. 2008. 